\title{
INSIGHTS
}

\section{Family reflections: a histiocytosis journey}

\author{
Tracy Brown ${ }^{1}$ \\ Pediatric Research (2019) 85:750-751; https://doi.org/10.1038/s41390-019-0302-2
}

It was dim in the room as I gently rocked my 16-month-old son lan, the light from the lamp casting a warm glow on the wispy fine blond hairs of his head. His warm body snuggled into mine, his bright blue eyes stared up at me, his sweet smile betrayed the battle going on inside. The battle between the rouge cells and the normal ones, the good and the bad-duking it out.

Just 3 weeks earlier he had been diagnosed with Langerhans cell Histiocytosis ( $\mathrm{LCH}$ ) of the lymph nodes, bone, and skin. Most of the bone lesions, where the bone had eroded from the disease, resided in his skull. As I sat there rocking my only child I imagined where the X-rays had shown the dark gray spots against the light gray of his skull, the darkness underscoring the void of bone. I gently kissed his head all over, wishing with each kiss to heal the holes in his skull. Willing the love in my heart to comingle with the chemo coursing through his veins and heal my baby.

It had been 5 months since we first noticed a swollen lymph node in lan's groin. Back then we met with the head of surgery at Children's National Medical Center in Washington, DC and were encouraged to watch and wait, as the likelihood of the swollen node being something bad was remote when compared to the risks of a sedated surgical biopsy at his age. We watched and waited and fretted for months, when finally with the encouragement of lan's pediatrician we opted to do the biopsy on 3 October 2006.

A week later we first heard the words Langerhans cell histiocytosis and our world narrowed to the sliver of the medical world reserved for rare diseases. I frantically searched the Internet and through the Histiocytosis Association (histio.org) learned that LCH is characterized by an over-abundance of white blood cells called histiocytes. These cells damage the surrounding organs, bone, and tissue in the form of lesions or tumors. At that time we learned that LCH primarily affects children under the age of 10, and in children under 2 was often fatal (thankfully in the last 12 years those stats have changed).

We learned that just 1 in 200,000 are afflicted by LCH-and our son was one of those children. It was devastating to not only discover were we dealing with a potentially fatal disease, but one so rare that it received little funding for research. In fact, few medical professionals were even familiar with it. In reading the message boards we soon realized how lucky we were to have received such a quick diagnosis and to live in an area where our son could be treated by doctors familiar with $\mathrm{LCH}$.

After a full workup of an MRI, CT scan, bone scan, bone marrow biopsy, and X-rays, we learned that in addition to the lymph nodes and slight case of LCH on his scalp, his bones were riddled with evidence of the disease. He had lesions in his cheek bones, hip bone, ribs, and skull-so very many lesions in his skull. I could not fathom how we did n't realize how sick he had been.
Thus we embarked on what we thought would be a 6-month journey into Rare Disease Land, but one that in the end would last nearly 5 years. lan's first treatment protocol consisted of a 6-week induction of weekly vinblastine and daily prednisone (LCH III protocol). After 6-weeks his oncologist opted to do another 6week induction before moving on to monthly maintenance in January 2007. We put our heads down and soldiered on through the treatment, confident it would work and he would beat the disease. He completed the vinblastine/prednisone maintenance in May 2007.

Two months later he was scanned and the disease had resurged with a vengeance, no parent wants to read a pathology report that states, "...the lesions in the skull are too numerous to count". There were also more lesions in his ribs and vertebra. So it was back to the drawing board and another treatment protocol. This time he did another 6-week induction of vinblastine with prednisone before moving on to 1 year of maintenance, which included monthly vinblastine and prednisone, plus daily $6 \mathrm{mp}$ and weekly Methotrexate. We found creative ways to entice our toddler to take his daily meds, and to keep our sanity when the steroid effects caused him to rage. This year was one of the hardest we faced as lan grew up at clinic thinking all kids got their "tubey" (port-a-catheter) accessed and had a favorite nurse. We spent more time at the ER than I care to remember, and were admitted several times for neutropenia. Even so, at the end of that 15 months of treatment we were cautious but optimistic that we had finally beat histio.

Three months later we were devastated to learn through scans that the disease was back. In truth, we feared it had returned because the lymph node behind lan's right ear had been swollen and the sunken look and dark circles under his eyes were signs we began to recognize meant the disease was back. This time, the multi-national team of doctors consulting on lan's case provided varying recommendations, but we ultimately decided on a one year protocol of vincristine, Ara-C, and prednisone. He would take daily prednisone for the entire year, while each cycle he'd receive vincristine and Ara-C on day 1 of treatment at clinic, and days 2-4 of treatment would consist of Ara-C administered at home. He started with 1 week on-1 week off, 1 week on-2 weeks off, 1 week on-3 weeks off, and so on throughout the year. Although by this point we had been living in Rare Disease Land for more than 2 years and practically had nursing degrees by proxy, neither my husband nor I were emotionally prepared for the task of administering that first home chemo to our son and deaccessing his port on Christmas Day. A chemo spill kit and toxic drugs in our fridge were not our idea of holiday cheer.

We thought for sure, after yet another year of treatment lan was going to beat it. But 3 months after finishing the Ara-C and

${ }^{1}$ Herndon, Virginia, USA

Correspondence: Tracy Brown (info@pedres.org)

Published online: 19 January 2019 
vincristine treatment we knew it was back, scans confirmed it in February 2010. We knew but the oncologist's confirmation still hit us hard. At that point we really only had one option left for treatment, 2Cda. This most toxic treatment consisted of 5 days of infusions followed by 2 weeks off. It was the hardest treatment yet on lan's little 4-year-old body; daily doses of neupogen 7-10 days after treatment, time after time of delayed treatment due to neutropenia, and exhaustion on all fronts from spending our lives at clinic and not at school, playgrounds, birthday parties, and family vacations.

We were thrown for a loop when part way through this treatment lan developed three new lesions. That had never occurred while he was on chemotherapy and we were dumbfounded at what it could mean. We were encouraged to finish treatment, yet in the end the three lesions remained and we were faced with the toughest decision of our lives. Did we try something else? Did we stop? Did we go back to the less toxic vinblastine and prednisone protocol? Once more the team of five LCH experts consulting on lan's case all provided differing opinions. After much thought and consideration of lan's health and quality of life, we opted to give his poor little body a break for 3 months. When he was finally scanned the lesions had improved, 3 months later they were better yet, and 3 months after that we had his port-a-cat removed.

Four and half years since his first chemo and we were finally done. During that time lan endured: 129 chemotherapy treatments, 440 doses of oral chemo, 1252 doses of steroids, 340X-rays, 15 sedations, 10 MRIs, 7 bone scans, 6 CT scans, 5 PET scans, 3 surgeries, 2 bone marrow biopsies, 2 fluoroscopies, 1 (awful) spinal tap, 1 laryngoscopy, 22 ER visits, 3 hospital stays, and thousands of doses of supporting drugs like antibiotics and antinausea medications. Countless blood draws, needle pokes, IV bags, urine tests, and finger pokes.

An accounting of the emotional toll remains harder to assess. Our son grew up thinking all of this was normal. He was subjected to treatment after toxic treatment because there just isn't enough known about LCH to ensure an effective treatment from the start. He received far more radiation in his first 5 years of life than most people ever receive. He missed out on the normal joys of childhood while we not only fought for his life, but raised awareness and funding for research throughout that fight.

Despite how grateful we are that he has been histio free for nearly 8 years, we are now faced with monitoring the long-term side effects of so many years of treatments and scans. Are the executive functioning challenges he faces a result of the drugs, the disease, or genetics? Will the cataracts in his eye ever grow worse? Will the hearing loss he suffered deteriorate further? Will the statistics on secondary cancers be his fate? Will the disease ever return?

Although it took many years of setbacks for lan to finally beat $\mathrm{LCH}$, we remain amazed and so grateful to the perseverance of the researchers who continue to dedicate their lives to unlocking the secrets of histio despite their own setbacks. It is our sincerest hope that funding research in the following areas could improve the quality of histio patient's lives: identification of more effective, less toxic treatments; better methods for assessing progress that rely on fewer radiological scans; and clear roadmaps for treatment when patient's are not responsive to first line medications. Thank you to the many researchers around the world who are actively seeking the answers to these tough questions. 